\title{
The Underlying Simplicity at the Subatomic Domain Can Describe the Diverse and Complex Universe - Where Matter and Energy are the Flipped Side of the Same Coin
}

\author{
Prasenjit Debnath
}

Designation: PhD Student Organization: NIT Agartala, India

\begin{abstract}
The Quantum theory deals with the microscopic structure of the Universe that describes the finer details of nature at the subatomic domain. To a physicist, quantum mechanics is one of the three great pillars supporting our intuition of evocative, bewildering and fascinating natural World as we continuously struggle with the inescapable strangeness of the subatomic domain as if something strange is always afoot. It is well accepted fact that quantum mechanics is a physical theory that describes the way things behave at subatomic level which is quite dazzling in its precision and explanatory power. A test of quantum electrodynamics, the oldest and best understood of the modern quantum theory that involves measuring the way an electron behaves in the vicinity of a magnetic force. Many of the technological and social changes that have revolutionized our lives have actually risen out of the fundamental theoretical research carried out with only motivation to understand the World in a better way than ever. For example, the invention of transistor actually came with the fundamental theoretical research on the Uncertainty Principle which states that the uncertainty of the position of a particle times the uncertainty of its velocity is always a constant. If we double the certainty of the position of the particle, we have to make half the certainty of its velocity and vice versa. Quantum theory is perhaps the prime example of the infinitely esoteric in the sense that it describes a World in which a particle really can be in several places at once and it moves from one place to another by exploring the entire Universe simultaneously- the theory of simultaneity. The diverse and complex Universe is actually made of a few tiny particles according to the rules of quantum theory which is so simple that can be summarized on the back of an envelope. The more we understand about the elemental nature of the Universe, the simpler it looks. We have already discovered that everything in the diverse and complex Universe is nothing but the different assemblies of atoms, and the wide variety of atoms are constructed using the three building blocks at the sub-atomic domain i.e. subatomic particles: protons, neutrons and electrons. We have also discovered that protons and neutrons ate made up of combination of three entities called quarks. And that is where things stop as far as recent discovery is concerned. But the recent progress in particle physics inspiring the quest for the ultimate unified theory of physics that can describe everything in the Universe in one go. The discovery of Boson particle certainty will revolutionaries the Newton's mass concept and gravity into whole new looks. To an optimistic point of view, we must hope the best out of the progress in physics.
\end{abstract}

Keyword: The quantum theory, Transistors and the Uncertainty Principle, Subatomic domain: protons, neutrons and electrons, Quarks, The Esoteric Universe.

\section{Introduction and Theory}

Our lives are actually a function of the physical time [1]. Many trillion molecules come together to form living being $[2,3]$ with a single and unique identity at a given particular time instant. It has something the other unique identity at another time instant. Thus, till the end of the living being [4, 5] (although the end is relative, I meant according to the psychological point of view what it generally means, whatever may be it is), it keeps getting new unique identities at different time instants with no two identities at two different time instants are identical to each other. At the present state of the Universe, the physical time is unidirectional $[6,7]$, the reason we cannot get back into old unique identities that we already left in earlier time instants $[8,9]$. So, unique identities are mere one way traffic for us $[10,11]$, once left, it is left for ever [12], at least within the psychologically limited point of view $[13,14]$. But certainly we are not esoteric as predicted in the Quantum Mechanics $[15,16]$ because we are not in the several places at once and we are not moving from one place to another place by exploring the entire Universe simultaneously which disprove the theory of simultaneity at the macroscopic scale of structure of the Universe. Because we are made of trillion particles [17, 18], as basic building blocks of us [19], the particles can never be esoteric either. More precisely, a particle can have a single and unique address or identity at a given particular time instant. And it can have something the other unique identity or address at another time instant only, which disproves the theory of simultaneity itself at the subatomic domain too. When we move, it is actually many trillion atoms or molecules are moving together [20, 21] which are bounded by electromagnetic force [22, 23]. Thus each atoms and molecules have their own unique identity in the composition as the living being $[24,25]$. But we ignore individual atom's or molecules' unique identity and rather we prefer the collective psychological unique identity as a living being that formed by the many trillion atoms or molecules together [26]. Why we have more psychological weight on the collective identity as living being over the individual atoms or molecules identity that formed the collective one, according to Charles Darwin, it is popularly called natural selection $[27,28]$. One particle one address at a time enhances the support of the theory of determinism. We actually live in preordained and predefined Universe where everything is deterministic; it does not matter it is past or future or something else, whatever may be. 


\section{International Journal of Science and Research (IJSR) \\ ISSN (Online): 2319-7064}

Index Copernicus Value (2013): 6.14 | Impact Factor (2014): 5.611

\section{On Matter and Energy}

Matter and energy are the flipped sides of the same coin. There are two critical masses $m_{1}$ and $m_{2} . m_{1}$ can be termed as the upper critical mass and $m_{2}$ can be termed as the lower critical mass. The upper critical mass $\left(m_{1}\right)$ discriminates between ordinary matters and the matters which display both particle and wave characteristics. The lower critical mass $\left(m_{2}\right)$ discriminates between the matters that display both particle-wave behavior and the matters which we call mass less energy or pure energy. Thus the particles below the lower critical mass $\left(m_{2}\right)$ shows pure interference behavior, we call them wave propagation which are evenly deposited everywhere, for example, bombarding of photons from the Sun are evenly deposited on the Earth. The masses between $m_{1}$ and $m_{2}$ are the transition masses which display both particle characteristics as well as wave characteristics such as electron deposition by the two slits on the screen in the Double-slit experiment. The results demonstrate the principle of wave-particle duality [29]. They can neither be termed as pure particle (ordinary matter) nor pure energy (wave propagation). As we come down from $m_{1}$ to $m_{2}$, the pure particle characteristics gradually decrease and pure energy characteristics (wave propagation) increase gradually. Above the critical mass $m_{1}$, the matters display a dominant ordinary particle characteristics and hidden or recessive energy characteristics. Whereas below mass $m_{2}$, the matter displays dominant wave characteristics and hidden or recessive matter characteristics. The masses between the critical masses $m_{1}$ and $m_{2}$ displays a mixture of partial matter and partial wave characteristics such as uneven deposition of electrons on the screen which is neither wave propagation not pure matter characteristics either, but a compromise between the two.

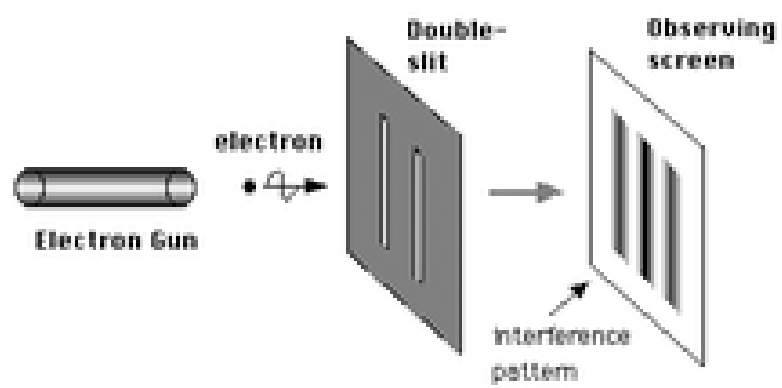

Figure 1: the interference pattern of electron on the screen travelled through double slit from the electron gun in the Double slit experiment
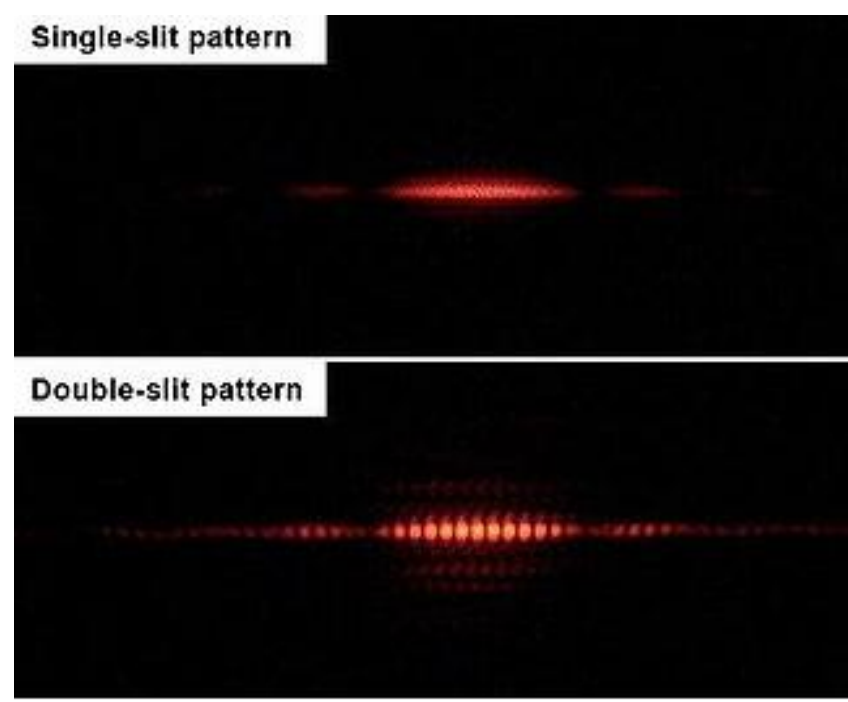

Figure 2: The interference pattern of electron on the screen travelled through the single slit and double slit from the electron gun
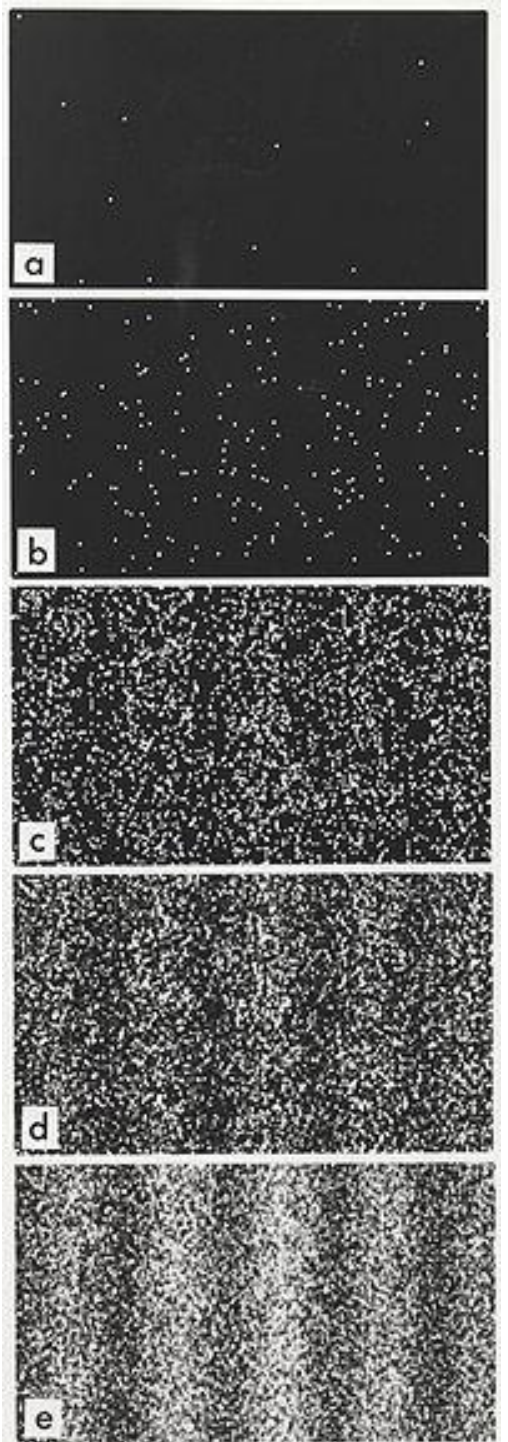

Figure 3: Electrons build up on the screen in the Double slit experiment

Ordinary matters (of masses $>m_{1}$ ) have only dominant particle characteristics and hidden wave characteristics like 


\section{International Journal of Science and Research (IJSR) \\ ISSN (Online): 2319-7064}

Index Copernicus Value (2013): 6.14 | Impact Factor (2014): 5.611

Earth movement can be only described by particle characteristics as it has very very negligible wave or interference characteristics (can be termed as hidden energy characteristics). The photon movement $\left(<m_{2}\right)$ has dominant wave characteristics and very very negligible matter characteristics (can be termed as hidden matter characteristics).

\section{What $E=m C^{2}$ DoES}

The famous Einstein's equation:

$$
E=m C^{2}
$$

Where $m$ is the mass of the body.

$C$ is the velocity of light $3,00,000$ kilometers/ second or $1,86,000$ miles per second.

$E$ is the energy.

The transformation of mass into energy in $E=m C^{2}$ is nothing but transformation of mass $\left(>m_{1}\right)$ to mass $\left(<m_{2}\right)$ and transformation of energy into mass is nothing but transformation of mass $\left(<m_{2}\right)$ to mass $\left(>m_{1}\right)$. On psychological recognition point of view, one display pure interference and the other display ordinary matters characteristics.

\section{Conclusion}

We do not live in an esoteric World rather we live in a deterministic World where everything is preordained and predefined. Energy or force is nothing but the act of particles $\left(<m_{2}\right)$ which we call virtual particles or mass less particles. If we go from higher mass to lower mass, a range of masses will display dominant ordinary matter characteristics and recessive wave characteristics which is very very negligible (hidden energy characteristics), when we pass the upper critical mass $m_{1}$, the masses will start interfering and particle characteristics are gradually replaced by wave characteristics as we come down from $m_{1}$ to $m_{2}$. When we will cross the lower critical mass $m_{2}$, wavy pattern becomes so dominant that particle characteristics becomes very very negligible which we can call as hidden particle characteristics which actually unifies matter with energythey are truly the flipped side of the same coin.

\section{Acknowledgment}

I cordially admire Dr. Aparna Nath, Associate Professor and my PhD Guide, The department of Physics, National Institute of Technology, Agartala, India, for the epitome of inspiration and motivation to write this particular paper with perfection and accuracy. I am extremely thankful to her from all possible help she made to write this paper. Also I am thankful to The Department of Physics of National Institute Of Technology Agartala (NIT Agartala) for proper conduct and coordination.

\section{References}

[1] Brian Cox, Jeff Forshaw, "The quantum universe: everything that can happen does happen", Penguin Books, pp. 1-44.

[2] Stephen Hawking, "A Briefer History of Time", Bantam Books, London, pp. 1-145.

[3] https://en.wikipedia.org/wiki/Double-slit_experiment

[4] http://map.gsfc.nasa.gov/universe/uni_fate.html

[5] Stephen Hawking, "Black holes and Baby Universes and other essays", Bantam Press, London 2013, ISBN 978-0-553-40663-4

[6] Stephen Hawking, "The Grand Design", Bantam Books, London 2011

[7] Stephen Hawking, "A Brief History of Time", Bantam Books, London 2011, pp. 156-157. ISBN-978-0-55310953-5

[8] Stephen Hawking, "The Universe in a Nutshell", Bantam Press, London 2013, pp. 58-61, 63, 82-85, 9094, 99, 196. ISBN 0-553-80202-X

[9] Stephen Hawking, "The Beginning of Time", A Lecture.

[10] Stephen Hawking, “Stephen Hawking's Universe: Strange Stuff Explained", PBS site on imaginary time.

[11] Stephen Hawking, "How to build a time machine", 27 April, 2010.

[12] Uno Ingard, K "Fundamental of Waves \& oscillations", Cambridge University Press. P. 38, ISBN-0-521-33957XOxford: The British Academy, 1999

[13]A. Zee, "Quantum Field Theory in a Nutshell", Princeton University Press, 2003

[14] Storrs McCall, "A Model of the Universe", Oxford: Clarendon Press, 1994

[15]Craig Callender, "Time, Reality and Experience", Cambridge, UK: Cambridge University Press.

[16]Craig Callender, "Thermodynamic Asymmetry in Time", The Stanford Encyclopedia of Philosophy (Spring 2002 Edition)

[17] Storrs McCall, "A Model of the Universe", Oxford: Clarendon Press, 1994

[18]Robin Le Poidevin and Murray McBeath, "The Philosophy of Time" Oxford: Oxford University Press, 1993

[19] Newton-Smith, W.H., "The Structure of Time". London: Routledge \& Kegan Paul, 1980.

[20]Barry Dainton, "Time and Space", Ithaca: McGillQueen's University Press, 2001

[21] Robin Le Poidevin, "Questions of Time and Tense", Oxford: Oxford University Press, 1998.

[22]Nerhlich, Graham, "What Spacetime Explains". Cambridge: Cambridge University Press, 1994.

[23] Sklar, Lawrence, "Space, Time, and Space-time". CA: University of California Press, 1974.

[24] Whitrow, G., "The Natural Philosophy of Time". Oxford: Oxford University Press, 1961. (2nd edn., 1980.)

[25] S.W. Hawking, and G.F.R. Ellis, "The Large Scale Structure of Space-Time", Cambridge University Press, (1973).

[26] Stephen Hawking, "A stubbornly persistent illusion-The essential scientific works of Albert Einstein", Running Press Book Publishers, Philadelphia, London 2011.

[27] Flynn, John L, "Time travel literature", on 29-09-2006 


\section{International Journal of Science and Research (IJSR) \\ ISSN (Online): 2319-7064}

Index Copernicus Value (2013): 6.14 | Impact Factor (2014): 5.611

[28] Stephen Hawking, "The Theory of Everything", Jaico Books, pp. 1-110.

[29] Stephen Hawking, “The Illustrated A Brief History of Time”, Bantam Books, pp. 1-21.

\section{Author Profile}

Prasenjit Debnath born in Agartala, Tripura, India on $15^{\text {th }}$ of March 1979 . He is pursuing a $\mathrm{PhD}$ degree in the Department of Physics in National Institute of Technology Agartala (NIT Agartala), India. 